\title{
Comparison of laryngoscopy and intubating conditions using kings vision laryngoscope and C-MAC video laryngoscope
}

\author{
Sarika M. Shetty ${ }^{1}$, Vyshnavi S., ${ }^{2, *}$, Dharanindra M. ${ }^{3}$, Archana KN ${ }^{4}$ \\ ${ }^{1,2}$ Assistant Professor, ${ }^{3}$ Junior Resident, ${ }^{4}$ Professor, JSS Medical College and Hospital, Mysore, Karnataka, India
}

*Corresponding Author:

Email: vyshgiri@gmail.com

Received: $07^{\text {th }}$ July, 2017

Accepted: $11^{\text {th }}$ December, 2017

\begin{abstract}
Introduction and Aims: Currently wide arrays of video laryngoscopes are available to facilitate laryngoscopy and endotracheal intubation in routine and difficult airway patients, intensive care units (ICU) and emergency settings. This study is undertaken to compare the efficacy of C-MAC and King Vision video laryngoscope for easy laryngoscopy and endotracheal intubation in patients with no predictors of difficult airway.

Materials and Methods: 60 adult patients undergoing elective surgery were randomly allocated into 2 groups for intubation using either King Vision laryngoscope (KVL group) or C-MAC video laryngoscope (C-MAC group). The parameters recorded were need for external laryngeal manipulation, percentage of glottis opening (POGO score), Cormack Lehane grading, number of attempts and time taken for successful endotracheal intubation and the airway morbidity. Systolic, diastolic, mean blood pressure and heart rate were measured preoperatively and at 1 and 2 min following endotracheal intubation in both the groups. The obtained data were analysed using chi-square test and Student's t-test using SPSS software

Results: The use of Kings Vision laryngoscope or CMAC video laryngoscope did not differ significantly with respect to good laryngoscopy and intubating conditions. But airway morbidity was significantly lower in the KVL group (10\%) when compared with the C-MAC group (40\%) p<0.015. Rise in systolic blood pressure(SBP) at $1 \mathrm{~min}$ following laryngoscopy was significantly less in KVL group(135.20 \pm 16.604$)$ than in C-MAC group $(142.00 \pm 10.055) \mathrm{p}<0.042$.

Conclusions: The use of C-MAC or KVL provided excellent laryngoscopy and intubating conditions except for the airway morbidity and haemodynamic stability which were better with the use of KVL.
\end{abstract}

Keywords: Video laryngoscopes, Endotracheal intubation, Airway morbidity, Haemodynamic changes.

\section{Introduction}

Video laryngoscopes have gained popularity in recent years as device which provide glottic view from a video-camera or video chip positioned close to the tip of video laryngoscope blade. These laryngoscopes are modified based on the needs of the anaesthesiologist and defined by the patient's airway anatomy, thus making them unique in their design. ${ }^{1}$ Each modification has its own advantages and disadvantages. Recent systematic review and meta analysis have shown that video laryngoscopes can offer better view of the glottic opening when compared to standard direct laryngoscopy and are better alternative options for management of difficult airways. ${ }^{2-4}$

C-MAC video laryngoscope [Karl Storz GmbH, Tuttlingen, Germany] has a Macintosh steel blade with no edges and gaps for hygienic traps and has the unique advantage of obtaining both direct laryngoscopic view and a camera view that is displayed on the video screen.

Kings vision video laryngoscope has an OLED display monitor which operates on the click of a button and is attached to the disposable blade, which can be of two types- a non channelled blade or a channelled blade.

There were no studies conducted in our institute comparing these two video laryngoscopes, hence this study was undertaken in our teaching hospital with the aim of comparing the two video layngoscopes with respect to optimum patient outcome and safe conduct of anesthesia.

\section{Materials and Methods}

After obtaining ethics committee approval, 60 adults patients in the age group of 18-60 yrs under American Soceity of Anaesthesiologists-physical status(ASA-PS) $<3$, with Mallampatti grade (MP) 1and 2 undergoing elective surgery were enrolled for the study. Exclusion criteria included history of difficult mask ventilation or intubation, presence of any predictors of difficult ventilation or intubation, presence of any airway pathology, pregnancy and morbidly obese patients(BMI>35).

A thorough pre-anaesthetic examination was done 24-48 hours prior to the surgery and all the co-morbid conditions, medications of the patient were noted and classified into appropriate ASA-PS grading. Height and weight of the patient were noted and BMI was calculated accordingly. Mallampatti grading of the airway was assessed as per standard protocol. Written informed consent was obtained from the patient, proper fasting guidelines were followed and appropriate preoperative medications were advised. By the computer generated randomization method, patients were randomly allocated into KVL group or C-MAC 
group and Kings Vision laryngoscope (KVL) or CMAC video laryngoscope (C-MAC) were used for laryngoscopy respectively.

On the day of surgery, patients were shifted to the Operating room, monitors such as electrocardiogram, non invasive blood pressure and saturation probe were connected and baseline parameters of systolic blood pressure (SBP), diastolic blood pressure (DBP), mean blood pressure (MBP), heart rate (HR) and oxygen saturation were recorded. Intravenous (IV) fluid infusion was started and premedications given were inj midazolam $0.5 \mathrm{mg}$ iv, inj glycopyrrolate $0.2 \mathrm{mg}$ iv, inj. ondansetron $4 \mathrm{mg}$ iv, inj fentanyl $2 \mu / \mathrm{kg}$ iv. As part of the preparation of the video laryngoscopes and the endotracheal tube (ETT) prior to induction, when KVL was used, appropriate sized lubricated ETT was channeled through the slot for the tube in the scope. When C-MAC was used, a malleable stylet in a hockey stick shape was passed through the appropriate sized ETT to obtain the perfect curvature of the tube.

Patients were preoxygenated for 3 min with $100 \%$ oxygen and induced with inj. propofol $2 \mathrm{mg} / \mathrm{kg}$ iv. Once the patients were apneic, adequacy of ventilation was checked by bag and mask ventilation, observing for adequate chest rise and end tidal carbon dioxide (etco2). Inj suxamethonium $1.5 \mathrm{mg} / \mathrm{kg}$ was used (as per the institutional protocol at the time of this study) to facilitate endotracheal intubation after which patients were ventilated for 60 secs. Laryngoscopy was done with either KVL in the KVL group or C-MAC in the CMAC group.

All laryngoscopies and endotracheal intubations were executed by an experienced anaesthesiologist (who has performed a minimum of 25 successful laryngoscopy and endotracheal intubation with both KVL and C-MAC video laryngoscope). On laryngoscopy with either of the scopes if glottic visualization was inadequate, an experienced second assistant was directed to give external laryngeal manipulation (BURP maneuver-backward, upward, rightward pressure) so as to align the glottis for a good visualization of the vocal cords and to facilitate endotracheal intubation. Visualization of scope monitor by the examiner provided an accurate assessment of POGO score and the Cormack Lehane grading. Successful intubation attempt was defined as an attempt in which the ETT was placed in the trachea as confirmed visually by the passage of the ETT through the glottis. More than two attempts if needed for successful intubation was considered as a failure and not included in our study. Then endotracheal intubation was achieved by conventional Macintosh laryngoscope, owing to increased ease of use and more experience with the use of this laryngoscope. Successful intubation time was defined as the time between blade insertion into the mouth and visually confirming the passage of the tube through the glottis.
Following laryngoscopy with either of the scopes, trauma and any amount of blood seen on the scope, lips, gums, orophanyx and tongue with or without breakage or trauma to the teeth was considered as airway morbidity. The detached tooth if any present in the oral cavity, was retrieved using a Magill's forceps. Firm pressure was applied if any bleeding was noticed.

Placement of ETT was confirmed later by bilateral chest auscultation, EtCO2 and the tube was secured after inflation of the tube cuff. Haemodynamic variables such as SBP, DBP, MBP and HR were documented at 1 and $2 \mathrm{~min}$ following endotracheal intubation.

Anaesthesia was maintained with inhalational agents, oxygen, nitrous oxide and muscle relaxants. At the end of the procedure patients were reversed and trachea was extubated, shifted to postoperative ward for further monitoring.

\section{Results}

60 patients with normal airway anatomy undergoing elective surgery were enrolled for this study in which Kings Vision laryngoscope $(\mathrm{n}=30)$ and $\mathrm{C}$ MAC video laryngoscope $(n=30)$ were used for laryngoscopy and intubation. The data obtained were tabulated and represented as absolute numbers with or without percentages or as mean \pm standard deviation (SD). For analysis of continuous variables independent samples t-test was applied and for categorical variables chi-square test was used. Value of $\mathrm{p}<0.05$ was considered significant in this study.

Patient characteristics such as age, sex and ASAPS grading were recorded and the demographic profile was compared between the two groups, which showed no statistically significant difference. BMI was calculated by recording the height and weight of the patient which was also similar in both the groups (Table 1). Mallampatti grading in both the groups showed no statistical difference.

Laryngoscopy and intubation characteristics of the patient in each of the group were recorded and compared and the values along with statistical analysis were obtained as in table 2. External laryngeal manipulation to improve the glottic visualization or for successful intubation was needed in 14 patients $(46.7 \%)$ in KVL group and in 19 patients (63.3\%) in C-MAC group which was not statistically significant (p0.194).After optimisation of the scope position and manual external manipulation of the glottis, Cormack Lehane grading was grade 1 or $2 \mathrm{a}$ in all the patients and POGO scoring was between the ranges of $95-100 \%$ in both the groups (table 4). In the KVL group, number of attempts required to successfully intubate the patients were equally distributed as $50 \%$ in first and second attempt. But in the C-MAC group, $12(40 \%)$ patients could be successfully intubated in the first attempt and $16(53.3 \%)$ in the second attempt. The remaining $2(6.7 \%)$ patients required more than two attempts for 
successful intubation, which was considered as a failure to intubate in our study. But statistical difference was insignificant (p-0.889) between the groups. The time required for successful intubation was 14.60+5.568 seconds in KVL group and $16.43+2.528$ seconds in CMAC group, and this difference was found to be statistically insignificant (p-0.462).

Airway morbidity as shown in table 3 was noted in both the groups that included trauma to the lips, tongue, oropharynx and breakage of the teeth. In the KVL group only $3(10 \%)$ patients were noted to have bleeding from the lips and oropharynx, where as in the C-MAC group $12(40 \%)$ had airway morbidity. Of these
12 patients with airway morbidity in the C-MAC group, three patients had breakage of teeth (expulsion of loose teeth) and nine patients had bleeding from the lips, tongue and oropharynx. Statistical analysis revealed significantly less incidence of airway trauma in KVL group (p-0.015)

Among the periodically measured haemodynamic parameters in both the groups, the rise in systolic blood pressure from the preoperative values to one min after intubation was significantly less in KVL group(135.20 \pm 16.604$)$ as compared to the C-MAC group $(142.00 \pm 10.055)$ with a $\mathrm{p}$ value of 0.042 as shown in table 5 .

Table 1: Mallampati Class \& BMI

\begin{tabular}{|c|c|c|c|}
\hline Mallampati class & KVL & C-MAC & P value \\
\hline 1 & $7(23.3 \%)$ & $12(40 \%)$ & \multirow{2}{*}{0.272} \\
\cline { 1 - 3 } & $23(76.7 \%)$ & $18(60 \%)$ & \\
\hline BMI $\left(\mathrm{kg} / \mathrm{m}^{2}\right)$ & $27.143 \pm 2.2589$ & $26.857 \pm 1.6192$ & 0.575 \\
\hline
\end{tabular}

Table 2: Number of Attempts \& Time Required for Intubation

\begin{tabular}{|c|c|c|c|}
\hline Number of Attempts & KVL & C-MAC & P value \\
\hline 1 & $15(50 \%)$ & $12(40 \%)$ & 0.889 \\
\hline 2 & $15(50 \%)$ & $16(53.3 \%)$ & \\
\hline $\begin{array}{l}\text { Time for intubation } \\
\text { (seconds) }\end{array}$ & $14.60 \pm 5.568$ & $16.43 \pm 2.528$ & 0.462 \\
\hline
\end{tabular}

Table 3: Airway Morbidity and External Laryngeal Manipulation

\begin{tabular}{|l|c|c|c|}
\hline & KVL & C-MAC & P value \\
\hline $\begin{array}{l}\text { External Laryngeal } \\
\text { Manipulation }\end{array}$ & $14(46.7 \%)$ & $19(63.3 \%)$ & 0.194 \\
\hline Airway morbidity & $3(10 \%)$ & $12(40 \%)$ & 0.015 \\
\hline
\end{tabular}

Table 4: POGO Score and Cormack Lehane grading

\begin{tabular}{|l|l|l|l|l|}
\hline & & KVL & C-MAC & p value \\
\hline POGO score (\%) & & $95-100 \%$ & $95-100 \%$ & \\
\hline $\begin{array}{l}\text { Cormack Lehane } \\
\text { grading }\end{array}$ & 1 & $27(90 \%)$ & $28(93.33 \%)$ & \multirow{2}{*}{0.23} \\
\cline { 2 - 4 } & $2 \mathrm{a}$ & $3(10 \%)$ & $2(6.66 \%)$ & \\
\hline
\end{tabular}

Table 5: Haemodynamic changes

\begin{tabular}{|c|c|c|c|c|}
\hline & & KVL & C-MAC & P value \\
\hline \multirow{3}{*}{$\begin{array}{l}\text { MBP } \\
(\mathrm{mm} \mathrm{Hg})\end{array}$} & Basal & $88.43 \pm 5.728$ & $88.47 \pm 9.712$ & 0.987 \\
\hline & 1 & $98.00 \pm 5.831$ & $96.30 \pm 12.180$ & 0.493 \\
\hline & 2 & $92.63 \pm 5.648$ & $91.73 \pm 8.263$ & 0.624 \\
\hline \multirow{3}{*}{$\begin{array}{l}\text { SBP } \\
(\mathrm{mm} \mathrm{Hg})\end{array}$} & Basal & $123.23 \pm 9.449$ & $124.23 \pm 16.498$ & 0.775 \\
\hline & 1 & $142.00 \pm 10.055$ & $135.20 \pm 16.604$ & 0.042 \\
\hline & 2 & $135.47 \pm 10.715$ & $130.13 \pm 12.331$ & 0.070 \\
\hline \multirow{3}{*}{$\begin{array}{l}\text { DBP } \\
(\mathrm{mm} \mathrm{Hg})\end{array}$} & Basal & $71.70 \pm 7.359$ & $71.17 \pm 8.682$ & 0.798 \\
\hline & 1 & $76.57 \pm 6.796$ & $77.37 \pm 12.425$ & 0.758 \\
\hline & 2 & $72.07 \pm 8.043$ & $73.03 \pm 8.739$ & 0.657 \\
\hline \multirow{3}{*}{$\begin{array}{l}\text { HR } \\
\text { (per } \\
\text { minute) }\end{array}$} & Basal & $76.47 \pm 12.428$ & $77.73 \pm 12.163$ & 0.691 \\
\hline & 1 & $85.40 \pm 11.134$ & $88.27 \pm 11.231$ & 0.325 \\
\hline & 2 & $81.53 \pm 9.968$ & $79.90 \pm 9.970$ & 0.528 \\
\hline
\end{tabular}




\section{Discussion}

Many studies have been conducted between the various video laryngoscopes and conventional laryngoscopes to study the intubating times, the success rate of intubation and the complications, however no studies have compared the two widely used video laryngoscopes i.e., C-MAC and KVL.

C-MAC videolaryngoscope is available in 3 sizes. It has a flattened slim blade profile with slanted edges to avoid damage to mouth and teeth. ${ }^{5}$ It is inserted using standard direct laryngoscopic technique. ${ }^{1,5}$ In contrast to many previous video laryngoscopes, the CMAC scope has the unique advantage of obtaining both direct laryngoscopic view and a camera view that is displayed on the video screen. This may be very helpful for educational purpose, because the instructor is able to follow and guide the student's laryngoscopy and intubation technique. Moreover, the very clear camera view may be stored as an image or video stream on a commercially available secure digital card and subsequently used for education or documentation.

Kings vision video laryngoscope has an OLED display monitor attached to the disposable blade. It offers \# 3 Macintosh blades which are of two types- a standard non channelled blade that requires the use of a stylet to direct the ETT through the laryngeal opening and another channelled blade where the ETT attains the shape of the preformed curvature of the channel which guides it through the vocal cords. The monitor is simple which operates on the click of a button (but without recording options) and the LED light at the tip of the blade gives good illumination to get a clear view of the glottis.

Video Laryngoscopes offer many advantages such as improved laryngeal visualization, less cervical spine movement, short learning curve, less force required as compared to direct laryngoscopy and higher success rate. The disadvantages include difficulty in passage of the ETT in spite of a good POGO score, fogging and secretions obscuring the view, loss of depth perception and different techniques of laryngoscopy and intubation with different types of video laryngoscope. ${ }^{6}$

In this present study patients were selected with no history and predictors of difficult ventilation or intubation. In order to use the video laryngoscopes effectively during anticipated or unanticipated difficult intubation it is essential that the user have enough experience using the scope in patients with a normal airway. Mallampatti grading was restricted to 1 and 2 in our study and morbidly obese patients with BMI of more than 35, which are a potential predictor of difficult airway, were not included. In a similar study ease and success of intubation was studied in a population with predicted difficult airway with C-MAC video laryngoscope and Macintosh laryngoscope and it was concluded that C-MAC laryngoscopy was a useful device for the initial approach to a potential difficult airway. ${ }^{7}$ During awake intubations using KVL and fibre scope in suspected difficult airway patients, KVL proved to be more beneficial. ${ }^{8}$ Awake fibreoptic intubation, which is the gold standard for anticipated difficult airway situations, requires the patient to be cooperative and the user to be an expert with the use of the fibre scope. These requirements of a successful fibre optic intubation are annulled by the use of video laryngoscopes as they are used in a manner similar to conventional laryngoscope in a well-sedated patient. In a study using video laryngoscopes in the emergency department, it was concluded that the Glide video laryngoscope and the C-MAC were associated with similar rates of intubation success. ${ }^{9}$

External laryngeal manipulation was defined as any external manual manipulation of the glottis intended to improve the laryngeal view or facilitate endotracheal tube passage. However in our study 14/30 in KVL group and 19/30 in C-MAC group required some manipulation, which was not statistically significant. Cormack Lehane $(90 \%$ grade 1 in KVL group and $93.3 \%$ grade 1 in CMAC group, p value0.23 , which is statistically insignificant.) and POGO score $(95-100 \%)$ were similar in both study groups. Requirement of optimisation maneuvers (BURP or second assistant) was least in the C-MAC video laryngoscope group in a similar study during intubation with conventional Macintosh and McCoy blades when compared with C-MAC video laryngoscopes and CMAC -D blades. ${ }^{10}$

In the same study, time taken for successful intubation was much less with the direct laryngoscopy which was attributed to the familiarity of the participants to those conventional scopes than to the newer video laryngoscopes. ${ }^{10}$ Similar study was undertaken which concluded that C-MAC required shorter intubation time and fewer intubation attempts when compared with McGrath video laryngoscopes in anticipated difficult airway patients. ${ }^{1}$ A study comparing the time required for successful intubation with Airtraq laryngoscope $(38 \pm 18$ secs $)$ and KVL $(26 \pm 11 \mathrm{secs})$ proved KVL to be more efficient. ${ }^{11}$ Similarly, intubation times were noted to be $16.9 \mathrm{secs}$ (8-60.0) with Macintosh, 20.5secs (7.2-60.0) with Kings Vision channelled scope and 60secs (11.0-60.0) with Kings Vision non channelled scope and it was concluded that KVL channelled scope was as advantageous as Macintosh but not non channelled scope for use by novice personnel. ${ }^{12}$ In our study following endotracheal intubation, the time to remove the malleable stylet from the ETT during C-MAC laryngoscopy and to separate the ETT from the channel of the KVL was not taken into account and so time for successful intubation was calculated as insertion of the scope blade into the mouth until the visual confirmation of passage of tube through the glottis which was statistically insignificant between the two groups. Shorter intubation time with minimal number of attempts of intubation is the goal of successful 
endotracheal intubation which prevents the patient from desaturating minimises trauma and maintains hemodynamic stability, all these advantages being highly significant in emergency conditions and in critically ill patients. Fewer the intubation attempts, lesser the hemodynamic response to intubation)

Airway morbidity as noticed by blood stains on the lips, tongue, oropharynx or trauma to the dentures is significantly seen in C-MAC group 12/30(40\%) as compared to the KVL group $3 / 30(10 \%) \mathrm{p}<0.015$. This can probably be explained by the fact that the endotracheal tube and the video laryngoscope were inserted as a single unit while using KVL, whereas the tube and the scope were inserted as two separate units while using CMAC, increasing the chances of inadvertent injuries in the process. In a study comparing Macintosh and C-MAC video laryngoscope minor tissue injury and bleeding in the oral cavity was noted in both the groups ${ }^{[13]}$. Similarly, in another study blood traces were noted in $6 \%$ of patients when C-MAC was used for laryngoscopy ${ }^{[14]}$ In our study the variation in systolic blood pressure was noted to be significant in $\mathrm{C}$ MAC group $(135.20 \pm 16.604)$ when compared to KVL group $(142.00 \pm 10.055) \mathrm{p}<0.042$ at $1 \mathrm{~min}$ following laryngoscopy. This is clinically significant when compared to the baseline blood pressure, especially in KVL group. This could be attributed to the increased incidence of airway trauma associated with the use of C-MAC scope when compared to the KVL scope in our study. However, the haemodynamic stability was achieved in the successive readings.

The limitation of this study is that the sample size was small. In recent times the video laryngoscopes are cost effective in the setting of a difficult airway for which previously a costlier fibreoptic bronchoscope or more invasive approaches like needle cricothyrotomy or tracheostomy were the other alternative technique for securing airway. Even though we have the options of supra glottic devices like LMA, combitube etc, in the setting of difficult airway, these videolaryngoscopes can be considered superior as they help to secure a definitive airway using the gold standard endotracheal tube under direct vision. In this study the use of both the video laryngoscopes is limited to patients with normal airway, However, its use in patients with anticipated and unanticipated difficult airway needs to be further evaluated reducing the need for alternative techniques like fibreoptic bronchoscopy.

\section{Conclusion}

Although KVL and C-MAC video laryngoscopes have been an efficient video laryngoscope in this study we conclude that KVL is a better alternative to C-MAC for laryngoscopy and endotracheal intubation in patients with normal airway.

\section{References}

1. Ng I, Hill AL, Williams DL, Lee K, Segal R. "Randomized controlled trial comparing the McGrath videolaryngoscope with the C-MAC videolaryngoscope in intubating adult patients with potential difficult airways." British journal of anaesthesia. (2012) 109,43943.

2. Niforopoulou P, Pantazopoulos I, Demestiha T, Koudouna E, Xanthos T. "Video-laryngoscopes in the adult airway management: a topical review of the literature." Acta Anaesthesiologica Scandinavica (2010) 54, 1050-61.

3. Su YC, Chen CC, Lee YK, Lee JY, Lin KJ. "Comparison of video laryngoscopes with direct laryngoscopy for tracheal intubation: a meta-analysis of randomised trials." European Journal of Anaesthesiology (2011) 28,788-95.

4. Griesdale DE, Liu D, McKinney J, Choi PT. "Glidescope ${ }^{\circledR}$ video-laryngoscopy versus direct laryngoscopy for endotracheal intubation: a systematic review and meta-analysis." Canadian Journal of Anesthesia/Journal canadiend'anesthésie (2012) 59, 4152.

5. Cavus E, Thee C, Moeller T, Kieckhaefer J, Doerges V, Wagner K. "A randomised, controlled crossover comparison of the C-MAC videolaryngoscope with direct laryngoscopy in 150 patients during routine induction of anaesthesia." BMC anesthesiology (2011)11,6.

6. Channa AB." Video laryngoscopes." Saudi journal of anaesthesia (2011) 5,357.

7. Aziz MF, Dillman D, Fu R, Brambrink AM. "Comparative effectiveness of the C-MAC video laryngoscope versus direct laryngoscopy in the setting of the predicted difficult airway." The Journal of the American Society of Anesthesiologists (2012) 116,62936.

8. Gaszynska E, Gaszynski T. "The King Vision" ${ }^{\mathrm{TM}}$ video laryngoscope for awake intubation: series of cases and literature review." Therapeutics and clinical risk management (2014) 10,475.

9. Mosier J, Chiu S, Patanwala AE, Sakles JC. “A comparison of the GlideScope video laryngoscope to the C-MAC video laryngoscope for intubation in the emergency department." Annals of emergency medicine (2013) 61,414-20.

10. Jain D, Dhankar M, Wig J, Jain A. "Comparison of the conventional C-MAC and the D-blade C-MAC with the direct laryngoscopes in simulated cervical spine injury-a manikin study". Brazilian Journal of Anesthesiology (English Edition) (2014) 64,269-74.

11. Ali QE, Amir SH, Jamil S, Ahmad S. "A comparative evaluation of the Airtraq and King Vision video laryngoscope as an intubating aid in adult patients." ActaanaesthesiologicaBelgica (2015) 66,81-5.

12. Akihisa Y, Maruyama K, Koyama Y, Yamada R, Ogura A, Andoh T. "Comparison of intubation performance between the King Vision and Macintosh laryngoscopes in novice personnel: a randomized, crossover manikin study." Journal of anesthesia (2014) 28,51-7.

13. Noppens RR, Geimer S, Eisel N, David M, Piepho T. "Endotracheal intubation using the C-MAC ${ }^{\circ}$ video laryngoscope or the Macintosh laryngoscope: a prospective, comparative study in the ICU" Critical Care (2012) 16,R103.

14. Piepho T, Fortmueller K, Heid FM, Schmidtmann I, Werner C, Noppens RR. "Performance of the C-MAC video laryngoscope in patients after a limited glottic view using Macintosh laryngoscopy." Anaesthesia (2011) $66,1101-5$. 